\title{
LINEAR DIFFERENTIAL EQUATIONS WITH UNBOUNDED DELAYS AND A FORCING TERM
}

\author{
JAN ČERMÁK AND PETR KUNDRÁT
}

Received 10 September 2002

The paper discusses the asymptotic behaviour of all solutions of the differential equation $\dot{y}(t)=-a(t) y(t)+\sum_{i=1}^{n} b_{i}(t) y\left(\tau_{i}(t)\right)+f(t), t \in I=\left[t_{0}, \infty\right)$, with a positive continuous function $a$, continuous functions $b_{i}, f$, and $n$ continuously differentiable unbounded lags. We establish conditions under which any solution $y$ of this equation can be estimated by means of a solution of an auxiliary functional equation with one unbounded lag. Moreover, some related questions concerning functional equations are discussed as well.

\section{Introduction}

In this paper, we study the problem of the asymptotic bounds of all solutions for the delay differential equation

$$
\dot{y}(t)=-a(t) y(t)+\sum_{i=1}^{n} b_{i}(t) y\left(\tau_{i}(t)\right)+f(t), \quad t \in I=\left[t_{0}, \infty\right),
$$

where $a$ is a positive continuous function on $I ; b_{i}, f$ are continuous functions on $I, \tau_{i}$ are continuously differentiable functions on $I$ fulfilling $\tau_{i}(t)<t, 0<\dot{\tau}_{i}(t) \leq \lambda_{i}<1$ for all $t \in I$ and $\tau_{i}(t) \rightarrow \infty$ as $t \rightarrow \infty, i=1, \ldots, n$.

The prototype of such equations may serve the equation with proportional delays

$$
\dot{y}(t)=-a y(t)+\sum_{i=1}^{n} b_{i} y\left(\lambda_{i} t\right)+f(t), \quad t \geq 0
$$

where $a>0, b_{i} \neq 0,0<\lambda_{i}<1, i=1, \ldots, n$, are real scalars. There are numerous interesting applications for (1.2) and its modifications, such as collection of current by the pantograph head of an electric locomotive, probability theory on algebraic structures or partition problems in number theory. Various special cases of (1.2) have been studied because of these applications, as well as for theoretical reasons (see, e.g., Bereketoglu and Pituk [1], Lim [11], Liu [12], or Ockendon and Taylor [15]). 
The study of these differential equations with proportional delays turned out to be the useful paradigm for the investigation of qualitative properties of differential equations with general unbounded lags. Some results of the above-cited papers have been generalized in this direction by Heard [7], Makay and Terjéki [13], and in [2, 3, 4]. For further related results on the asymptotic behaviour of solutions, see, for example, Diblík $[5,6]$, Iserles [8], or Krisztin [9].

In this paper, we combine standard methods from the theory of functional differential equations and some results of the theory of functional equations and difference equations to analyze the asymptotic properties of all solutions of (1.1). The main results are formulated in Sections 3 and 4. In Section 3, we derive the asymptotic estimate of all solutions of (1.1). Section 4 discusses some particular cases of (1.1) and improves the above derived estimate for these special cases. Both sections also present the illustrating examples involving, among others, (1.2).

\section{Preliminaries}

Let $t_{-1}:=\min \left\{\tau_{i}\left(t_{0}\right), i=1,2, \ldots, n\right\}$ and $I_{-1}:=\left[t_{-1}, \infty\right)$. By a solution of $(1.1)$, we understand a real-valued function $y \in C\left(I_{-1}\right) \cap C^{1}(I)$ such that $y$ satisfies (1.1) on $I$.

In the sequel, we introduce the notion of embeddability of given functions into an iteration group. This property will be imposed on the set of delays $\left\{\tau_{1}, \ldots, \tau_{n}\right\}$ throughout next sections.

Definition 2.1. Let $\psi \in C^{1}\left(I_{-1}\right), \dot{\psi}>0$ on $I_{-1}$. Say that $\left\{\tau_{1}, \ldots, \tau_{n}\right\}$ can be embedded into an iteration group $[\psi]$ if for any $\tau_{i}$ there exists a constant $d_{i}$ such that

$$
\tau_{i}(t)=\psi^{-1}\left(\psi(t)-d_{i}\right), \quad t \in I
$$

Remark 2.2. The problem of embeddability of given functions $\left\{\tau_{1}, \ldots, \tau_{n}\right\}$ into an iteration group $[\psi]$ is closely related to the existence of a common solution $\psi$ to the system of the simultaneous Abel equations

$$
\psi\left(\tau_{i}(t)\right)=\psi(t)-d_{i}, \quad t \in I, i=1, \ldots, n
$$

The complete solution of these problems have been described by Neuman [14] and Zdun [16]. These papers contain conditions under which (2.1) holds for any $\tau_{i}, i=1, \ldots, n$ (see also [10, Theorem 9.4.1]). We only note that the most important necessary condition is commutativity of any pair $\tau_{i}, \tau_{j}, i, j=1, \ldots, n$. Notice also, that if $\tau_{i}$ are delays, then $d_{i}$ must be positive.

\section{The asymptotic bound of all solutions of (1.1)}

The aim of this section is to formulate and prove the asymptotic estimate of all solutions of (1.1). We assume that all the assumptions imposed on $a, b_{i}, \tau_{i}$, and $f$ in Section 1 are valid. 
Theorem 3.1. Let $\left\{\tau_{1}, \ldots, \tau_{n}\right\}$ be embedded into an iteration group $[\psi]$. Let $y$ be a solution of (1.1), where $a(t) \geq K / \exp \{\alpha \psi(t)\}, 0<\sum_{i=1}^{n}\left|b_{i}(t)\right| \leq M a(t)$ for all $t \in I$ and suitable real constants $K>0, M>0, \alpha<1$. If $f(t)=O(\exp \{\beta \psi(t)\})$ as $t \rightarrow \infty$ for a suitable real $\beta$, then

$$
y(t)=O(\exp \{\gamma \psi(t)\}) \quad \text { as } t \longrightarrow \infty, \gamma>\max \left(\alpha+\beta, \frac{\log M}{d_{1}}, \ldots, \frac{\log M}{d_{n}}\right)
$$

where $d_{i}, i=1, \ldots, n$, are given by (2.1).

Proof. The substitution

$$
s=\psi(t), \quad z(s)=\exp \{-\gamma \psi(t)\} y(t)
$$

transforms (1.1) into the form

$$
\begin{aligned}
z^{\prime}(s)= & -\left[a(h(s)) h^{\prime}(s)+\gamma\right] z(s)+\sum_{i=1}^{n} b_{i}(h(s)) \exp \left\{-\gamma d_{i}\right\} h^{\prime}(s) z\left(\mu_{i}(s)\right) \\
& +f(h(s)) \exp \{-\gamma s\} h^{\prime}(s),
\end{aligned}
$$

where "'” stands for $d / d s, h(s)=\psi^{-1}(s)$, and $\mu_{i}(s)=\psi\left(\tau_{i}(h(s))\right)=s-d_{i}$ on $\psi(I), i=$ $1, \ldots, n$. This form can be rewritten as

$$
\begin{aligned}
& \frac{d}{d s}\left[\exp \left\{\gamma s+\int_{s_{0}}^{h(s)} a(u) d u\right\} z(s)\right] \\
& =\sum_{i=1}^{n} b_{i}(h(s)) \exp \left\{-\gamma d_{i}\right\} h^{\prime}(s) \exp \left\{\gamma s+\int_{s_{0}}^{h(s)} a(u) d u\right\} z\left(s-d_{i}\right) \\
& \quad+\exp \left\{\gamma s+\int_{s_{0}}^{h(s)} a(u) d u\right\} f(h(s)) \exp \{-\gamma s\} h^{\prime}(s),
\end{aligned}
$$

where $s_{0} \in \psi(I)$ is such that $\gamma+a(h(s)) h^{\prime}(s)>0$ for all $s \geq s_{0}$.

Put $\delta:=\min \left(d_{1}, \ldots, d_{n}\right)>0, s_{k}:=s_{0}+k \delta, J_{k}:=\left[s_{k-1}, s_{k}\right], k=1,2, \ldots$ Let $s^{*} \in J_{k+1}$. The integration of (3.4) over $\left[s_{k}, s^{*}\right]$ yields

$$
\begin{aligned}
\exp \{\gamma s & \left.+\int_{s_{0}}^{h(s)} a(u) d u\right\}\left.z(s)\right|_{s_{k}} ^{s^{*}} \\
= & \sum_{i=1}^{n} \int_{s_{k}}^{s^{*}} b_{i}(h(s)) \exp \left\{-\gamma d_{i}\right\} h^{\prime}(s) \exp \left\{\gamma s+\int_{s_{0}}^{h(s)} a(u) d u\right\} z\left(s-d_{i}\right) d s \\
& +\int_{s_{k}}^{s^{*}} \exp \left\{\gamma s+\int_{s_{0}}^{h(s)} a(u) d u\right\} f(h(s)) \exp \{-\gamma s\} h^{\prime}(s) d s
\end{aligned}
$$


that is,

$$
\begin{aligned}
z\left(s^{*}\right)= & \exp \left\{\gamma\left(s_{k}-s^{*}\right)-\int_{h\left(s_{k}\right)}^{h\left(s^{*}\right)} a(u) d u\right\} z\left(s_{k}\right) \\
& +\exp \left\{-\int_{s_{0}}^{h\left(s^{*}\right)} a(u) d u-\gamma s^{*}\right\} \\
& \times \sum_{i=1}^{n} \int_{s_{k}}^{s^{*}} b_{i}(h(s)) \exp \left\{-\gamma d_{i}\right\} h^{\prime}(s) \exp \left\{\gamma s+\int_{s_{0}}^{h(s)} a(u) d u\right\} z\left(s-d_{i}\right) d s \\
& +\exp \left\{-\int_{s_{0}}^{h\left(s^{*}\right)} a(u) d u-\gamma s^{*}\right\} \\
& \times \int_{s_{k}}^{s^{*}} \exp \left\{\gamma s+\int_{s_{0}}^{h(s)} a(u) d u\right\} f(h(s)) \exp \{-\gamma s\} h^{\prime}(s) d s .
\end{aligned}
$$

Put $M_{k}:=\sup \left\{|z(s)|, s \in \cup_{p=1}^{k} J_{p}\right\}, k=1,2, \ldots$ Then one can estimate $z\left(s^{*}\right)$ as

$$
\begin{aligned}
\left|z\left(s^{*}\right)\right| \leq & M_{k} \exp \left\{\gamma\left(s_{k}-s^{*}\right)-\int_{h\left(s_{k}\right)}^{h\left(s^{*}\right)} a(u) d u\right\} \\
& +M_{k} \exp \left\{-\int_{s_{0}}^{h\left(s^{*}\right)} a(u) d u-\gamma s^{*}\right\} \\
& \times \int_{s_{k}}^{s^{*}} \sum_{i=1}^{n}\left|b_{i}(h(s))\right| \exp \left\{-\gamma d_{i}\right\} h^{\prime}(s) \exp \left\{\gamma s+\int_{s_{0}}^{h(s)} a(u) d u\right\} d s \\
& +\exp \left\{-\int_{s_{0}}^{h\left(s^{*}\right)} a(u) d u-\gamma s^{*}\right\} \\
& \times \int_{s_{k}}^{s^{*}} \exp \left\{\gamma s+\int_{s_{0}}^{h(s)} a(u) d u\right\}|f(h(s))| \exp \{-\gamma s\} h^{\prime}(s) d s .
\end{aligned}
$$

Noting that

$$
\begin{gathered}
\sum_{i=1}^{n}\left|b_{i}(h(s))\right| \exp \left\{-\gamma d_{i}\right\} \leq M \exp \left\{-\gamma d_{i}\right\} a(h(s))<a(h(s)), \\
|f(h(s))| \exp \{-\gamma s\} \leq K_{1} \exp \{(\beta-\gamma) s\}, \quad K_{1}>0
\end{gathered}
$$

we can rewrite (3.7) as

$$
\begin{aligned}
\left|z\left(s^{*}\right)\right| \leq & M_{k} \exp \left\{\gamma\left(s_{k}-s^{*}\right)-\int_{h\left(s_{k}\right)}^{h\left(s^{*}\right)} a(u) d u\right\} \\
& +M_{k} \exp \left\{-\int_{s_{0}}^{h\left(s^{*}\right)} a(u) d u-\gamma s^{*}\right\} \\
& \times \int_{s_{k}}^{s^{*}} a(h(s)) h^{\prime}(s) \exp \left\{\gamma s+\int_{s_{0}}^{h(s)} a(u) d u\right\} d s
\end{aligned}
$$




$$
\begin{aligned}
& +K_{1} \exp \left\{-\int_{s_{0}}^{h\left(s^{*}\right)} a(u) d u-\gamma s^{*}\right\} \\
& \times \int_{s_{k}}^{s^{*}} \exp \left\{\gamma s+\int_{s_{0}}^{h(s)} a(u) d u\right\} h^{\prime}(s) \exp \{(\beta-\gamma) s\} d s .
\end{aligned}
$$

From here, we get

$$
\begin{aligned}
\left|z\left(s^{*}\right)\right| \leq & M_{k} \exp \left\{\gamma\left(s_{k}-s^{*}\right)-\int_{h\left(s_{k}\right)}^{h\left(s^{*}\right)} a(u) d u\right\} \\
& +\left(M_{k}+K_{2} \exp \left\{(\alpha+\beta-\gamma) s_{k}\right\}\right) \exp \left\{-\int_{s_{0}}^{h\left(s^{*}\right)} a(u) d u-\gamma s^{*}\right\} \\
& \times \int_{s_{k}}^{s^{*}} a(h(s)) h^{\prime}(s) \exp \left\{\gamma s+\int_{s_{0}}^{h(s)} a(u) d u\right\} d s
\end{aligned}
$$

where $K_{2}=K_{1} / K$. Using the assumptions imposed on $a$ and $\dot{\tau}_{i}$, we can estimate the inte$\operatorname{gral} I:=\int_{s_{k}}^{s^{*}} a(h(s)) h^{\prime}(s) \exp \left\{\gamma s+\int_{s_{0}}^{h(s)} a(u) d u\right\} d s$ as

$$
I \leq\left.\exp \left\{\gamma s+\int_{s_{0}}^{h(s)} a(u) d u\right\}\right|_{s_{k}} ^{s^{*}}\left(1+K_{3} e^{-\omega s_{k}}\right), \quad K_{3}>0, \omega=1-\alpha>0
$$

(for a similar situation see also [4]). Hence,

$$
\begin{aligned}
\left|z\left(s^{*}\right)\right| \leq & M_{k} \exp \left\{\gamma\left(s_{k}-s^{*}\right)-\int_{h\left(s_{k}\right)}^{h\left(s^{*}\right)} a(u) d u\right\} \\
& +\left(M_{k}+K_{2} \exp \left\{(\alpha+\beta-\gamma) s_{k}\right\}\right) \exp \left\{-\int_{s_{0}}^{h\left(s^{*}\right)} a(u) d u-\gamma s^{*}\right\} \\
& \times\left.\exp \left\{\gamma s+\int_{s_{0}}^{h(s)} a(u) d u\right\}\right|_{s_{k}} ^{s^{*}}\left(1+K_{3} \exp \left\{-\omega s_{k}\right\}\right) \\
\leq & M_{k}\left(1+K_{3} \exp \left\{-\omega s_{k}\right\}\right)+K_{2} \exp \left\{(\alpha+\beta-\gamma) s_{k}\right\}\left(1+K_{3} \exp \left\{-\omega s_{k}\right\}\right) \\
\leq & M_{k}^{*}\left(1+N \exp \left\{-\kappa s_{k}\right\}\right),
\end{aligned}
$$

where $M_{k}^{*}:=\max \left(M_{k}, K_{2}\right), \kappa:=\min (\omega, \gamma-\alpha-\beta)>0$, and $N>0$ is a constant large enough. Since $s^{*} \in J_{k+1}$ was arbitrary,

$$
M_{k+1}^{*} \leq M_{k}^{*}\left(1+N \exp \left\{-\kappa s_{k}\right\}\right) \leq M_{1}^{*} \prod_{j=1}^{k}\left(1+N \exp \left\{-\kappa s_{j}\right\}\right) .
$$

Now, the boundedness of $\left(M_{k}^{*}\right)$ as $k \rightarrow \infty$ implies via substitution (3.2) the asymptotic estimate (3.1).

Remark 3.2. This remark concerns the possible extension of our results to differential equations with delays intersecting the identity at the initial point $t_{0}$. These equations 
form a wide and natural class of delay differential equations (see the following examples) and have many applications (some of them have been mentioned in Section 1). Since we are interested in the behaviour at infinity, it is obvious that the main notions and results of this paper can be easily reformulated to this case.

Example 3.3. Consider the equation

$$
\dot{y}(t)=-(a+c \exp \{-t\}) y(t)+\sum_{i=1}^{n} b_{i} y\left(\lambda_{i} t\right)+f(t), \quad t \geq 0,
$$

where $a>0, c \geq 0, b_{i} \neq 0,0<\lambda_{i}<1, i=1, \ldots, n$ are constants and $f \in C([0, \infty))$ fulfils $f(t)=O\left(t^{\beta}\right)$ as $t \rightarrow \infty$. Let $\psi(t)=\log t$, then functions $\left\{\lambda_{1} t, \ldots, \lambda_{n} t\right\}$ can be embedded into an iteration group $[\psi]$. Indeed,

$$
\psi\left(\lambda_{i} t\right)=\psi(t)-\log \lambda_{i}^{-1}, \quad t>0, i=1, \ldots, n
$$

Then, by Theorem 3.1, the estimate

$$
y(t)=O\left(t^{\gamma}\right) \quad \text { as } t \longrightarrow \infty, \gamma>\max \left(\beta, \frac{\log \sum_{i=1}^{n}\left|b_{i}\right| / a}{\log \lambda_{1}^{-1}}, \ldots, \frac{\log \sum_{i=1}^{n}\left|b_{i}\right| / a}{\log \lambda_{n}^{-1}}\right)
$$

holds for any solution $y$ of (3.14).

\section{Some particular cases of (1.1)}

In this section, we first consider (1.1) in the homogeneous form

$$
\dot{y}(t)=-a(t) y(t)+\sum_{i=1}^{n} b_{i}(t) y\left(\tau_{i}(t)\right), \quad t \in I
$$

Using a simple modification of the proof of Theorem 3.1, we improve the conclusion of this theorem for the case of (4.1). We assume that all the assumptions of Theorem 3.1 are valid (the assumptions on $f$ are missing, of course). Using the same notation as in Theorem 3.1, we have the following theorem.

TheORem 4.1. Let $y$ be a solution of (4.1). Then

$$
y(t)=O(\exp \{\gamma \psi(t)\}) \quad \text { as } t \longrightarrow \infty, \gamma=\max \left(\frac{\log M}{d_{1}}, \ldots, \frac{\log M}{d_{n}}\right)
$$

Proof. Following the proof of Theorem 3.1, we can see that the condition on $\gamma$ in (3.1) becomes

$$
\gamma>\max \left(\frac{\log M}{d_{1}}, \ldots, \frac{\log M}{d_{n}}\right)
$$


in view of $f \equiv 0$ on $I$. Moreover, the inequality (3.8) can be replaced by

$$
\sum_{i=1}^{n}\left|b_{i}(h(s))\right| \exp \left\{-\gamma d_{i}\right\} \leq a(h(s)),
$$

and this implies the validity of (4.2).

Now, we consider (1.1) in another special form

$$
\dot{y}(t)=-a y(t)+b y(\tau(t))+f(t), \quad t \in I,
$$

where $a>0, b \neq 0$ are constants, $\tau \in C^{1}(I), \tau(t)<t, 0<\dot{\tau}(t) \leq \lambda<1$ for all $t \in I, \tau(t) \rightarrow \infty$ as $t \rightarrow \infty$, and $f \in C(I)$ fulfils $f(t)=O(\exp \{\beta \psi(t)\})$ as $t \rightarrow \infty$. Under these assumptions on $\tau$, there exists a function $\psi \in C^{1}(I), \dot{\psi}>0$ on $I$ such that

$$
\psi(\tau(t))=\psi(t)-\log \lambda^{-1}, \quad t \in I
$$

(for this and related results concerning (4.6) see, e.g., [10]). Then applying Theorem 3.1 to $(4.5)$, we can easily deduce that the property

$$
y(t)=O(\exp \{\gamma \psi(t)\}) \quad \text { as } t \longrightarrow \infty, \gamma>\max \left(\alpha+\beta, \frac{\log (|b| / a)}{\log \lambda^{-1}}\right)
$$

holds for any solution $y$ of (4.5).

The asymptotic behaviour of (4.5) has been studied in [3]. If we put

$$
\sigma:=\frac{\log (|b| / a)}{\log \lambda^{-1}}
$$

then using the previous notation we can recall the following result.

Theorem 4.2 [3, Theorem 2.3]. Consider (4.5), where $a>0, b \neq 0$ are constants, $\tau, f \in$ $C^{1}(I), \tau(t)<t, 0<\dot{\tau}(t) \leq \lambda<1$ for all $t \in I, \tau(t) \rightarrow \infty, f(t)=O(\exp \{\beta \psi(t)\})$, and $\dot{f}(t)=$ $O(\exp \{(\beta-1) \psi(t)\})$ as $t \rightarrow \infty$. If $y$ is a solution of (4.5), then

$$
y(t)= \begin{cases}O(\exp \{\sigma \psi(t)\}) & \text { as } t \longrightarrow \infty \text { if } \beta<\sigma, \\ O(\exp \{\sigma \psi(t)\} \psi(t)) & \text { as } t \longrightarrow \infty \text { if } \beta=\sigma, \\ O(\exp \{\beta \psi(t)\}) & \text { as } t \longrightarrow \infty \text { if } \beta>\sigma .\end{cases}
$$

It is easy to see that relations (4.9) yield sharper estimates of solutions than (4.7). On the other hand, we emphasize that the proof technique used in [3] is effective just for (4.5) and cannot be applied to more general equation (1.1). In the final part of this paper, we propose a simple way on how to extend the conclusions of Theorem 4.2 to some equation (4.5) with nonconstant coefficients. To explain the main idea, we consider (4.5), where the delayed argument is a power function.

Example 4.3. We consider the delay equation

$$
\dot{y}(t)=-a y(t)+b y\left(t^{\lambda}\right)+f(t), \quad t \geq 1,
$$


where $a>0, b \neq 0,0<\lambda<1$ are constants and $f \in C^{1}([1, \infty))$ fulfils the properties

$$
f(t)=O\left((\log t)^{\beta}\right), \quad \dot{f}(t)=O\left((\log t)^{\beta-1}\right) \quad \text { as } t \longrightarrow \infty .
$$

The corresponding Abel equation (4.6) has the form

$$
\psi\left(t^{\lambda}\right)=\psi(t)-\log \lambda^{-1}, \quad t \geq 1,
$$

and admits the function $\psi(t)=\log \log t$ as a solution with the required properties. Substituting this $\psi$ into assumptions and conclusions of Theorem 4.2, we obtain the following result, where $\sigma$ is given by (4.8), if $y$ is a solution of (4.10), then

$$
y(t)= \begin{cases}O\left((\log t)^{\sigma}\right) & \text { as } t \longrightarrow \infty \text { if } \beta<\sigma \\ O\left((\log t)^{\sigma} \log \log t\right) & \text { as } t \longrightarrow \infty \text { if } \beta=\sigma \\ O\left((\log t)^{\beta}\right) & \text { as } t \longrightarrow \infty \text { if } \beta>\sigma .\end{cases}
$$

Now, we consider the equation

$$
\dot{y}(t)=-\frac{a}{t} y(t)+\frac{b}{t} y\left(t^{\lambda}\right)+f(t), \quad t \geq 1,
$$

where $a, b$, and $\lambda$ are the same as above and $f \in C^{1}([1, \infty))$.

Setting

$$
s=\log t, \quad z(s)=y(t),
$$

we can convert (4.14) into the form

$$
z^{\prime}(s)=-a z(s)+b z(\lambda s)+f(\exp \{s\}) \exp \{s\}, \quad s \geq 0 .
$$

Now if the forcing term in (4.16) fulfils the required asymptotic properties, then applying Theorem 4.2 to (4.16) and substituting this back into (4.15), we get that relations (4.13) are valid for any solution $y$ of (4.14).

Remark 4.4. Following Example 4.3, we can extend asymptotic estimates (4.13) also to some other equations of the form

$$
\dot{y}(t)=-\dot{\varphi}(t)\left[a y(t)-b y\left(t^{\lambda}\right)\right]+f(t), \quad t \geq 1,
$$

where $\varphi \in C^{1}([1, \infty))$ and $\dot{\varphi}>0$ on $[1, \infty)$. If we introduce the change of variables

$$
s=\varphi(t), \quad z(s)=y(t)
$$

then (4.17) can be transformed into

$$
z^{\prime}(s)=-a z(s)+b z(\mu(s))+f\left(\varphi^{-1}(s)\right)\left(\varphi^{-1}\right)^{\prime}(s),
$$

where $\mu(s)=\varphi\left(\left(\varphi^{-1}(s)\right)^{\lambda}\right), s \in \varphi(I)$. Now if the delayed argument and the forcing term in (4.19) fulfil the assumptions of Theorem 4.2, then we can apply this theorem to (4.19) and via substituting (4.18) obtain the validity of (4.13) for any solution $y$ of (4.17). 


\section{Acknowledgment}

This research was supported by Grant 201/01/0079 of the Czech Grant Agency.

\section{References}

[1] H. Bereketoğlu and M. Pituk, Asymptotic constancy for nonhomogeneous linear differential equations with unbounded delays, Discrete Contin. Dyn. Syst. (2003), no. suppl., 100-107.

[2] J. Čermák, Asymptotic behaviour of solutions of some differential equations with an unbounded delay, E. J. Qualitative Theory of Diff. Equa., Proc. 6th Coll. Qualitative Theory of Diff. Equ., No.2, (2000), pp. 1-8.

[3] - A change of variables in the asymptotic theory of differential equations with unbounded delays, J. Comput. Appl. Math. 143 (2002), no. 1, 81-93.

[4] - The asymptotic of solutions for a class of delay differential equations, Rocky Mountain J. Math 33 (2003), no. 3, 775-786.

[5] J. Diblík, Asymptotic representation of solutions of equation $\dot{y}(t)=\beta(t)[y(t)-y(t-\tau(t))]$, J. Math. Anal. Appl. 217 (1998), no. 1, 200-215.

[6] A criterion for convergence of solutions of homogeneous delay linear differential equations, Ann. Polon. Math. 72 (1999), no. 2, 115-130.

[7] M. L. Heard, A change of variables for functional differential equations, J. Differential Equations 18 (1975), 1-10.

[8] A. Iserles, On the generalized pantograph functional-differential equation, European J. Appl. Math. 4 (1993), no. 1, 1-38.

[9] T. Krisztin, A note on the convergence of the solutions of a linear functional-differential equation, J. Math. Anal. Appl. 145 (1990), no. 1, 17-25.

[10] M. Kuczma, B. Choczewski, and R. Ger, Iterative Functional Equations, Encyclopedia of Mathematics and its Applications, vol. 32, Cambridge University Press, Cambridge, 1990.

[11] E.-B. Lim, Asymptotic bounds of solutions of the functional differential equation $x^{\prime}(t)=a x(\lambda t)+$ $b x(t)+f(t), 0<\lambda<1$, SIAM J. Math. Anal. 9 (1978), no. 5, 915-920.

[12] Y. Liu, Asymptotic behaviour of functional-differential equations with proportional time delays, European J. Appl. Math. 7 (1996), no. 1, 11-30.

[13] G. Makay and J. Terjéki, On the asymptotic behavior of the pantograph equations, Electron. J. Qual. Theory Differ. Equ. (1998), no. 2, 1-12.

[14] F. Neuman, Simultaneous solutions of a system of Abel equations and differential equations with several deviations, Czechoslovak Math. J. 32(107) (1982), no. 3, 488-494.

[15] J. R. Ockendon and A. B. Taylor, The dynamics of a current collection system for an electric locomotive, Proc. Roy. Soc. London Ser. A 322 (1971), 447-468.

[16] M. C. Zdun, On simultaneous Abel's equations, Aequationes Math. 38 (1989), no. 2-3, 163-177.

Jan Čermák: Institute of Mathematics, Brno University of Technology, Technická 2, 61669 Brno, Czech Republic

E-mail address: cermakh@mat.fme.vutbr.cz

Petr Kundrát: Institute of Mathematics, Brno University of Technology, Technická 2, 61669 Brno, Czech Republic

E-mail address: kundratp@mat.fme.vutbr.cz 


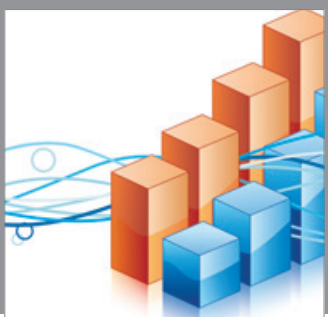

Advances in

Operations Research

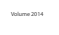

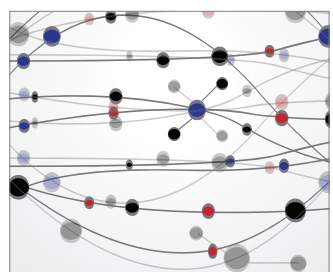

\section{The Scientific} World Journal
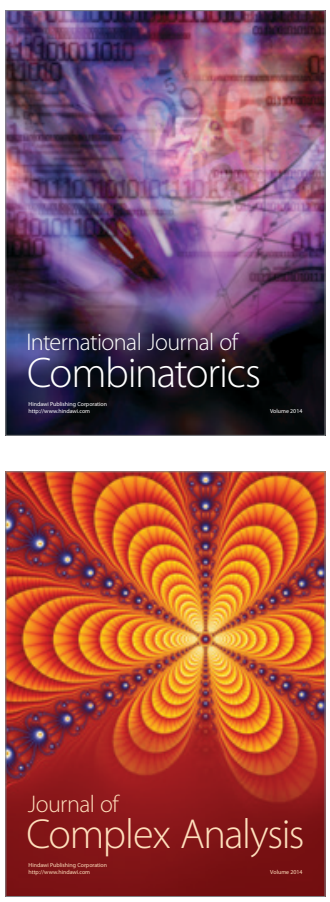

International Journal of

Mathematics and

Mathematical

Sciences
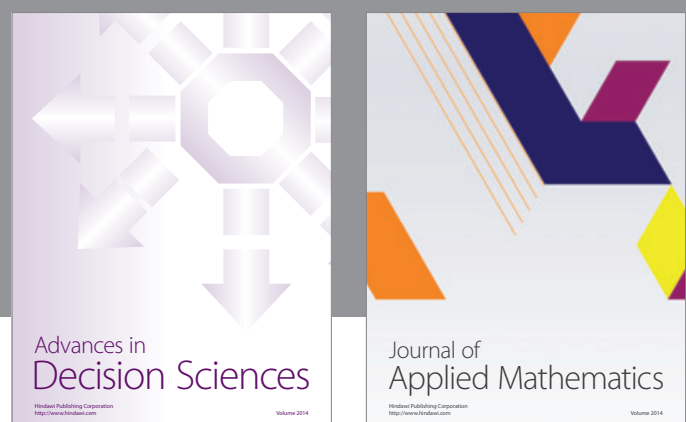

Journal of

Applied Mathematics
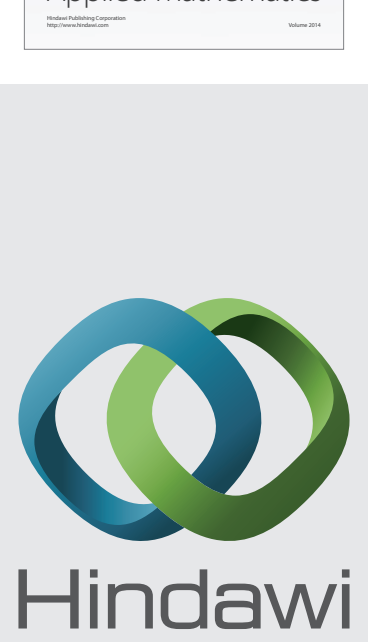

Submit your manuscripts at http://www.hindawi.com
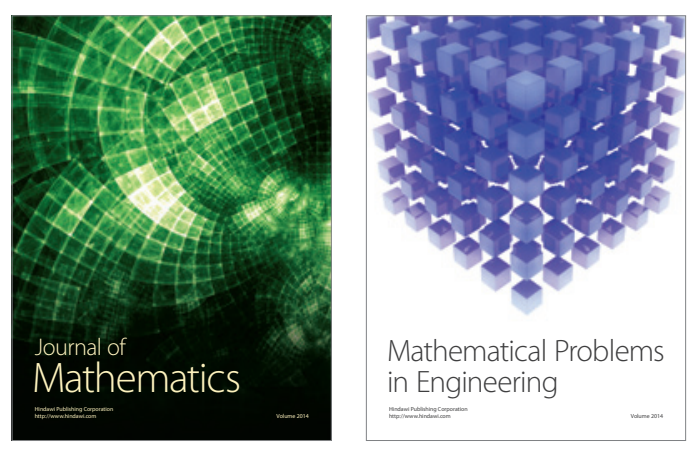

Mathematical Problems in Engineering
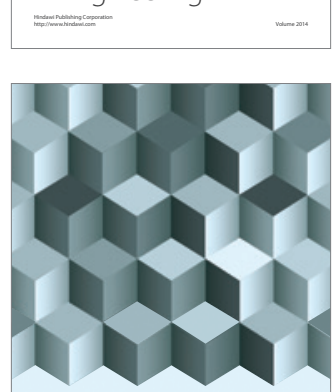

Journal of

Function Spaces
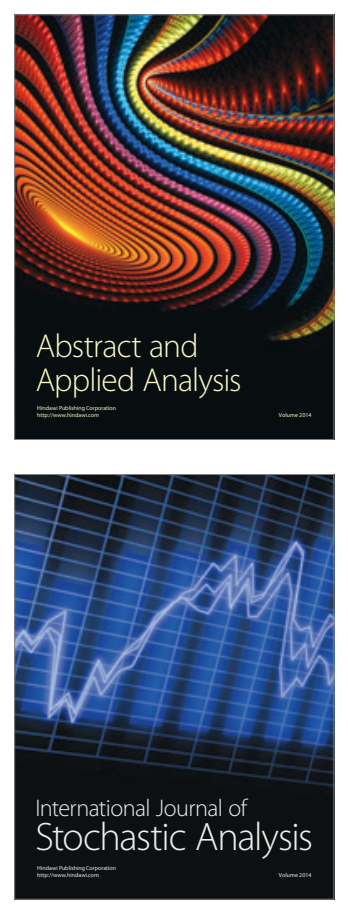

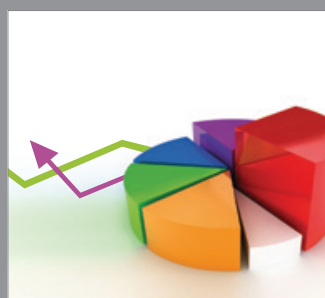

ournal of

Probability and Statistics

Promensencen
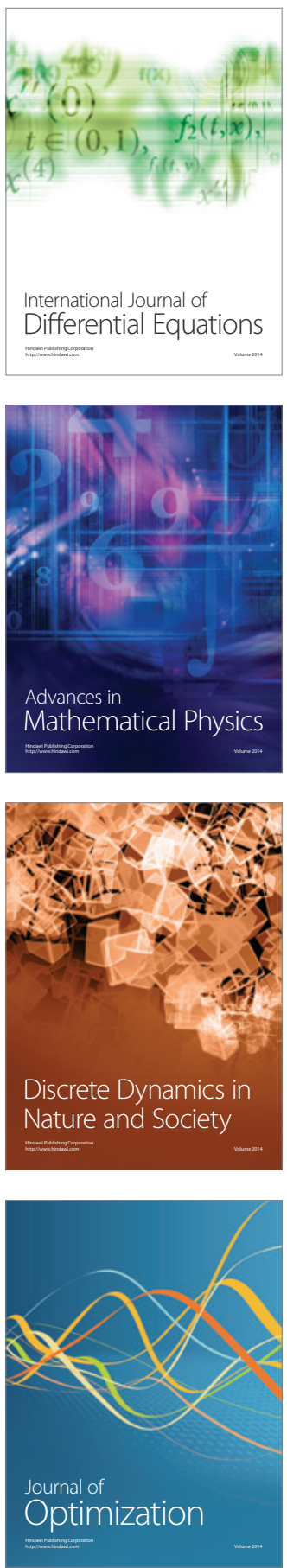